\title{
Structural stability for a Brinkman-Forchheimer type model with temperature-dependent solubility
}

\section{Wenhui Chen and Yan Liu*}

"Correspondence:

liuyan99021324@tom.com Department of Applied

Mathematics, Guangdong University of Finance, Guangzhou, 510521, P.R. China

\section{Springer}

\begin{abstract}
We study the structural stability for the Brinkman-Forchheimer equations with temperature-dependent solubility. We prove both the convergence and continuous dependence results for the Forchheimer coefficient $\lambda$. We also demonstrate how to get the same results for the Forchheimer equations.
\end{abstract}

MSC: 35B30; 35K55; 35Q35

Keywords: structural stability; Forchheimer coefficient; convergence result; continuous dependence

\section{Introduction}

In the last few years, some researchers have studied the question of the continuous dependence or convergence of solutions of problems in partial differential equations on the coefficients in the equations. It is called the question of structural stability. For one thing, when we study continuous dependence or convergence, the notion of structural stability is on changes in the model itself instead of the original data. The majority of references to work of this nature are given in the monograph of Ames and Straughan [1], which studies the structural stability about changes in the model itself. Hence, we tend to know that changes in the coefficients in the partial differential equations may be reflected physically by changes in the constitutive parameters. If we deeply study these equations by mathematical analysis, it is certainly giving us a helping hand to indicate their applicability in physics. For another, because of some inevitable errors, which may have occurred, continuous dependence or convergence results are significant. It is relevant to know the magnitude of the effect of such errors in the solutions. Consequently, we think it is valuable for us to study the subject of structural stability.

We tend to find a wide range of papers in the literature coping with the structural stability for varieties equations. Most of them focus on the Brinkman, Darcym, and Forchheimer models. These equations are discussed in the books of Nield and Bejan [2] and Straughan $[3,4]$. In addition, several papers have dealt with Saint-Venant type spatial decay results for the Brinkman, Darcy, Forchheimer, and other equations for porous media. More recent work on the stability and continuous dependence questions in porous media problems has been carried out by Ames and Payne [5], Franchi and Straughan [6], Kaloni and Qin

(c) 2016 Chen and Liu. This article is distributed under the terms of the Creative Commons Attribution 4.0 International License (http://creativecommons.org/licenses/by/4.0/), which permits unrestricted use, distribution, and reproduction in any medium, provided you give appropriate credit to the original author(s) and the source, provide a link to the Creative Commons license, and indicate if changes were made. 
[7], Kaloni and Guo [8], Payne and Straughan [9-11], Payne et al. [12], Lin and Payne [1315], Li and Lin [16], Celebi et al. [17, 18], Straughan [19], Scott [20], Scott and Straughan [21], and Harfash [22-24]. The fundamental model we study is based upon the equations of balance of momentum, balance of mass, conservation of energy, and conservation of salt concentration, adopting a Forchheimer approximation in the body force term in the momentum equation (see $[3,25])$,

$$
\left\{\begin{array}{l}
\frac{\partial u_{i}}{\partial t}+\lambda|u| u_{i}=-p_{, i}+\Delta u_{i}+g_{i} T-h_{i} C, \quad(x, t) \in \Omega \times[0, \tau] \\
\frac{\partial u_{i}}{\partial x_{i}}=0, \quad(x, t) \in \Omega \times[0, \tau], \\
\frac{\partial T}{\partial t}+u_{i} \frac{\partial T}{\partial x_{i}}=\Delta T, \quad(x, t) \in \Omega \times[0, \tau] \\
\frac{\partial C}{\partial t}+u_{i} \frac{\partial C}{\partial x_{i}}=\Delta C+L f(T)-k C, \quad(x, t) \in \Omega \times[0, \tau]
\end{array}\right.
$$

where $u_{i}$ is the velocity, $p$ denotes the pressure, $T$ is the temperature, and $C$ is the salt concentration. Here $g_{i}(x), h_{i}(x)$ are gravity fields. Here also $\Delta$ is the Laplacian operator. $a, b$, $L$, and $k$ are positive constants. Equations (1.1) follow in practice by employing a Forchheimer approximation which accounts for the variable $C$ allowing the incompressibility condition to hold (see Fife [26]). The function $f$ is at least $C^{1}$.

Equations (1.1) hold in the region $\Omega \times[0, \tau]$, where $\Omega$ is a bounded, simply connected, and star-shaped domain with boundary $\partial \Omega$ in $R^{3}$, and $\tau$ is a given number satisfying $0 \leq$ $\tau<\infty$. Associated with (1.1), we impose the boundary conditions

$$
u_{i}=0, \quad \frac{\partial T}{\partial n}=0, \quad \frac{\partial C}{\partial n}=0, \quad(x, t) \in \partial \Omega \times[0, \tau],
$$

and additionally the concentration is given at $t=0$, i.e.,

$$
\begin{aligned}
& u_{i}(x, 0)=u_{i 0}(x), \\
& T(x, 0)=T_{0}(x), \quad C(x, 0)=C_{0}(x), \quad x \in \Omega .
\end{aligned}
$$

We will derive both the convergence result and continuous result on the Forchheimer coefficient $\lambda$. In the present paper, a comma is used to indicate partial differentiation and the differentiation with respect to the direction $x_{k}$ is denoted as , $k$, thus $u_{, i}$ denotes $\frac{\partial u}{\partial x_{i}}$. The usual summation convection is employed with repeated Latin subscripts summed from 1 to 3 . Hence, $u_{i, i}=\sum_{i=1}^{3} \frac{\partial u_{i}}{\partial x_{i}},\|\cdot\|$ denotes the norm of $L^{2}$, and $\|\cdot\|_{p}$ denotes the norm of $L^{p}$.

\section{A priori bounds for the temperature $T$ and the salt concentration $C$}

Now we want an a priori bound or a maximum principle for $T$. Therefore multiplying $(1.1)_{3}$ by $T^{2 p-1}$ and integrating by parts, we can obtain

$$
\int_{\Omega} T^{2 p} d x-\int_{\Omega} T_{0}^{2 p} d x=-\frac{2(2 p-1)}{p} \int_{0}^{t} \int_{\Omega}\left(T^{p}\right)_{, i}\left(T^{p}\right)_{, i} d x d \eta \leq 0
$$

Inequality (2.1) is now integrated and then we take the $\frac{1}{2 p}$ power to find

$$
\left(\int_{0}^{t} \int_{\Omega} T^{2 p} d x d \eta\right)^{\frac{1}{2 p}} \leq\left(\int_{0}^{t} \int_{\Omega} T_{0}^{2 p} d x d \eta\right)^{\frac{1}{2 p}}
$$


We let $p \rightarrow \infty$ and then (2.2) leads to

$$
\sup _{[0, t]}\|T\|_{\infty} \leq\left\|T_{0}\right\|_{\infty}=T^{M} .
$$

Since $f$ is a $C^{1}$ function, and $T$ is bounded, we can easily see that there exists a constant $d$ such that

$$
f(T) \leq d
$$

For some $\xi \in\left(T, T^{*}\right)$, we easily get

$$
\left|f^{\prime}(\xi)\right| \leq k_{1}
$$

where $k_{1}$ is a positive constant.

Similarly we have

$$
\left(\int_{0}^{t} \int_{\Omega} C^{2 p} d x d \eta\right)^{\frac{1}{2 p}} \leq\left(\int_{0}^{t} \int_{\Omega} e^{(2 p-1)(t-\eta)}\left(k(p)+\int_{\Omega} C_{0}^{2 p} d x\right) d x d \eta\right)^{\frac{1}{2 p}}
$$

where $k(p)=\int_{0}^{\tau} \int_{\Omega}(L f(T))^{2 p} d x d t$.

We let $p \rightarrow \infty$, (2.6) leads to

$$
\sup _{[0, t]}\|C\|_{\infty} \leq C^{M},
$$

where $C^{M}=\max \left\{e^{\tau}\left\|C_{0}\right\|_{\infty}, L d e^{\tau}\right\}$

\section{Convergence and continuous dependence results for the Forchheimer coefficient $\lambda$}

Now, let $\left(u_{i}, T, C, p\right)$ be a solution to the boundary initial-value problem for the BrinkmanForchheimer model,

$$
\begin{aligned}
& \left\{\begin{array}{l}
\frac{\partial u_{i}}{\partial t}+\lambda|u| u_{i}=-p, i+\Delta u_{i}+g_{i} T-h_{i} C, \quad(x, t) \in \Omega \times[0, \tau], \\
\frac{\partial u_{i}}{\partial x_{i}}=0, \quad(x, t) \in \Omega \times[0, \tau], \\
\frac{\partial T}{\partial t}+u_{i} \frac{\partial T}{\partial x_{i}}=\Delta T, \quad(x, t) \in \Omega \times[0, \tau], \\
\frac{\partial C}{\partial t}+u_{i} \frac{\partial C}{\partial x_{i}}=\Delta C+L f(T)-k C, \quad(x, t) \in \Omega \times[0, \tau],
\end{array}\right. \\
& u_{i}=0, \quad \frac{\partial T}{\partial n}=0, \quad \frac{\partial C}{\partial n}=0, \quad(x, t) \in \partial \Omega \times[0, \tau], \\
& u_{i}(x, 0)=u_{i 0}(x), \\
& T(x, 0)=T_{0}(x), \quad C(x, 0)=C_{0}(x), \quad x \in \Omega .
\end{aligned}
$$

Moreover, let $\left(u_{i}^{*}, T^{*}, C^{*}, p^{*}\right)$ be a solution to the corresponding model with $\lambda=0$,

$$
\left\{\begin{array}{l}
\frac{\partial u_{i}^{*}}{\partial t}=-p_{, i}^{*}+\Delta u_{i}^{*}+g_{i} T^{*}-h_{i} C^{*}, \quad(x, t) \in \Omega \times[0, \tau], \\
\frac{\partial u_{i}^{*}}{\partial x_{i}}=0, \quad(x, t) \in \Omega \times[0, \tau], \\
\frac{\partial T^{*}}{\partial t}+u_{i}^{*} \frac{\partial T^{*}}{\partial x_{i}}=\Delta T^{*}, \quad(x, t) \in \Omega \times[0, \tau], \\
\frac{\partial C^{*}}{\partial t}+u_{i}^{*} \frac{\partial C^{*}}{\partial x_{i}}=\Delta C^{*}+L f\left(T^{*}\right)-k C^{*}, \quad(x, t) \in \Omega \times[0, \tau],
\end{array}\right.
$$




$$
\begin{aligned}
& u_{i}^{*}=0, \quad \frac{\partial T^{*}}{\partial n}=0, \quad \frac{\partial C^{*}}{\partial n}=0, \quad(x, t) \in \partial \Omega \times[0, \tau], \\
& u_{i}^{*}(x, 0)=u_{i 0}(x), \\
& T^{*}(x, 0)=T_{0}(x), \quad C^{*}(x, 0)=C_{0}(x), \quad x \in \Omega .
\end{aligned}
$$

The object of this section is to demonstrate that the solution of (3.1) converges to the solution of (3.5) as $\lambda \rightarrow 0$. Now, we define the difference variables $\omega_{i}, \pi, \theta$, and $S$ by

$$
\omega_{i}=u_{i}-u_{i}^{*}, \quad \pi=p-p^{*}, \quad \theta=T-T^{*}, \quad S=C-C^{*} .
$$

Then $\left(\omega_{i}, \theta, S, \pi\right)$ is a solution of the problem

$$
\left\{\begin{array}{l}
\frac{\partial \omega_{i}}{\partial t}+\lambda|u| u_{i}=-\pi_{, i}+\Delta \omega_{i}+g_{i} \theta-h_{i} S, \quad(x, t) \in \Omega \times[0, \tau] \\
\frac{\partial \omega_{i}}{\partial x_{i}}=0, \quad(x, t) \in \Omega \times[0, \tau], \\
\frac{\partial \theta}{\partial t}+\omega_{i} \frac{\partial T}{\partial x_{i}}+u_{i}^{*} \frac{\partial \theta}{\partial x_{i}}=\Delta \theta, \quad(x, t) \in \Omega \times[0, \tau], \\
\frac{\partial S}{\partial t}+\omega_{i} \frac{\partial C}{\partial x_{i}}+u_{i}^{*} S_{, i}=\Delta S+L\left(f(T)-f\left(T^{*}\right)\right)-k S, \quad(x, t) \in \Omega \times[0, \tau]
\end{array}\right.
$$

in $\Omega \times[0, \tau]$, subject to the boundary and initial conditions

$$
\begin{aligned}
& \omega_{i}=0, \quad \frac{\partial \theta}{\partial n}=0, \quad \frac{\partial S}{\partial n}=0, \quad(x, t) \in \partial \Omega \times[0, \tau], \\
& \omega_{i}(x, 0)=0, \\
& \theta(x, 0)=0, \quad S(x, 0)=0, \quad x \in \Omega .
\end{aligned}
$$

We will obtain the following result.

Theorem 1 Let $\left(u_{i}, T, C, p\right)$ be the classical solution to the initial-boundary problem (3.1)(3.4), $\left(u_{i}^{*}, T^{*}, C^{*}, p^{*}\right)$ be the classical solution to the initial-boundary problem (3.5)-(3.8) in $\Omega \times[0, \tau]$, and $\left(w_{i}, \theta, S, \pi\right)$ be the difference of $\left(u_{i}, T, C, p\right)$ and $\left(u_{i}^{*}, T^{*}, C^{*}, p^{*}\right)$, then the solution $\left(u_{i}, T, C, p\right)$ converges to the solution $\left(u_{i}^{*}, T^{*}, C^{*}, p^{*}\right)$ as the Boussinesq coefficient $\lambda$ tends to 0 . The difference $\left(w_{i}, \theta, S, \pi\right)$ satisfies

$$
\|\omega\|^{2}+\|\theta\|^{2}+\|S\|^{2} \leq \frac{2 \lambda}{3 M_{1}}\left(\frac{3}{4} k_{2}^{2} k_{3}^{\frac{1}{2}} k_{4}^{\frac{3}{2}}+\frac{1}{4}|\Omega|\right) e^{M_{1} \tau} .
$$

Proof We multiply (3.10) by $\omega_{i}$ and integrate over $\Omega$ to find

$$
\begin{aligned}
\frac{d}{d t}\|\omega\|^{2} & =-2 \lambda \int_{\Omega}|u| u_{i} \omega_{i} d x-2\|\nabla \omega\|^{2}+2 \int_{\Omega} g_{i} \theta \omega_{i} d x-2 \int_{\Omega} h_{i} S \omega_{i} d x \\
& \leq-2 \lambda \int_{\Omega}|u| u_{i} u_{i} d x+2 \lambda \int_{\Omega}|u| u_{i} u_{i}^{*} d x+2\|\omega\|^{2}+h^{2}\|S\|^{2}+g^{2}\|\theta\|^{2} \\
& \leq \frac{2}{3} \lambda \int_{\Omega}\left|u^{*}\right| u_{i}^{*} u_{i}^{*} d x+2\|\omega\|^{2}+h^{2}\|S\|^{2}+g^{2}\|\theta\|^{2} .
\end{aligned}
$$

We will use the following inequality in three dimensions:

$$
\|f\|_{4} \leq k_{2}^{\frac{1}{2}}\|f\|^{\frac{1}{4}}\|\nabla f\|^{\frac{3}{4}},
$$

where $k_{2}$ is a positive constant. 
From the above inequality and the Young inequality, we can get

$$
\begin{aligned}
\int_{\Omega}\left|u^{*}\right| u_{i}^{*} u_{i}^{*} d x & \leq \frac{3}{4} \int_{\Omega}\left(u_{i}^{*} u_{i}^{*}\right)^{2} d x+\int_{\Omega} \frac{1}{4} d x \\
& \leq \frac{3}{4} k_{2}^{2}\left\|u^{*}\right\|\left\|\nabla u^{*}\right\|^{3}+\frac{1}{4}|\Omega|,
\end{aligned}
$$

where $|\Omega|$ is the measure of $\Omega$.

We multiply (3.5) $)_{1}$ by $u_{i}^{*}$ and integrate over $\Omega$ to find

$$
\begin{aligned}
\frac{1}{2} \frac{d}{d t}\left\|u^{*}\right\|^{2}+\left\|\nabla u^{*}\right\|^{2}= & 7-\int_{\Omega} p_{, i}^{*} u_{i}^{*} d x+\int_{\Omega} g_{i} T^{*} u_{i}^{*} d x-\int_{\Omega} h_{i} C^{*} u_{i}^{*} d x \\
\leq & \left(g^{2} \int_{\Omega} u_{i}^{*} u_{i}^{*} d x \int_{\Omega}\left(T^{*}\right)^{2} d x\right)^{\frac{1}{2}} \\
& +\left(h^{2} \int_{\Omega} u_{i}^{*} u_{i}^{*} d x \int_{\Omega}\left(C^{*}\right)^{2} d x\right)^{\frac{1}{2}} \\
\leq & \left\|u^{*}\right\|^{2}+\frac{1}{2}|\Omega|\left[g^{2}\left(T^{M}\right)^{2}+h^{2}\left(C^{M}\right)^{2}\right],
\end{aligned}
$$

where $g^{2}=\max _{\Omega} g_{i} g_{i}, h^{2}=\max _{\Omega} h_{i} h_{i}$.

Integration of (3.18) leads to

$$
\left\|u^{*}\right\|^{2} \leq\left\|u_{0}\right\|^{2} e^{2 \tau}+\frac{1}{2}\left[g^{2}\left(T^{M}\right)^{2}+h^{2}\left(C^{M}\right)^{2}\right]|\Omega|\left(e^{2 \tau}-1\right)=k_{3} .
$$

We now want to give a bound for $\left\|\nabla u^{*}\right\|^{2}$. Multiplying (3.5) $)_{1}$ by $u_{i, t}^{*}$ and integrating over $\Omega$, we have

$$
\int_{\Omega} u_{i, t}^{*} u_{i, t}^{*} d x-\int_{\Omega} u_{i, t}^{*} u_{i, j j}^{*} d x=\int_{\Omega} g_{i} u_{i, t}^{*} T^{*} d x-\int_{\Omega} h_{i} u_{i, t}^{*} C^{*} d x
$$

We can obtain

$$
\begin{aligned}
& \int_{0}^{t} \int_{\Omega} u_{i, t}^{*} u_{i, t}^{*} d x d \eta+\left.\frac{1}{2} \int_{\Omega} u_{i, j}^{*} u_{i, j}^{*} d x\right|_{\eta=t} \\
& \leq \int_{0}^{t} \int_{\Omega} u_{i, t}^{*} u_{i, t}^{*} d x d \eta+\frac{1}{2} \int_{0}^{t} \int_{\Omega}\left(T^{*}\right)^{2} d x d \eta \\
& \quad+\frac{1}{2} \int_{0}^{t} \int_{\Omega}\left(C^{*}\right)^{2} d x d \eta+\frac{1}{2} \int_{\Omega} u_{i 0, j} u_{i 0, j} d x
\end{aligned}
$$

or

$$
\left.\int_{\Omega} u_{i, j}^{*} u_{i, j}^{*} d x\right|_{\eta=t} \leq\left(T^{M}\right)^{2}|\Omega| \tau+\left(C^{M}\right)^{2}|\Omega| \tau+\int_{\Omega} u_{i 0, j} u_{i 0, j} d x=k_{4} .
$$

Combining (3.15), (3.17), (3.19), and (3.22) gives

$$
\frac{d}{d t}\|\omega\|^{2} \leq \frac{2}{3} \lambda\left(\frac{3}{4} k_{2}^{2} k_{3}^{\frac{1}{2}} k_{4}^{\frac{3}{2}}+\frac{1}{4}|\Omega|\right)+2\|\omega\|^{2}+h^{2}\|S\|^{2}+g^{2}\|\theta\|^{2}
$$


Multiplying (3.10) 3 by $\theta$ and integrating over $\Omega$, we derive

$$
\begin{aligned}
\frac{d}{d t}\|\theta\|^{2}+2 \int_{\Omega} \theta_{, i} \theta_{, i} d x & =-2 \int_{\Omega} \theta \omega_{i} T_{, i} d x=2 \int_{\Omega} \theta_{, i} \omega_{i} T d x \\
& \leq 2 \int_{\Omega} \theta_{, i} \theta_{, i} d x+\frac{\left(T^{M}\right)^{2}}{2}\|\omega\|^{2} .
\end{aligned}
$$

The Lagrange theorem says that

$$
f(T)-f\left(T^{*}\right)=\theta f^{\prime}(\xi) .
$$

Multiplying (3.10) ${ }_{4}$ by $S$ and integrating over $\Omega$ and using (2.5), we find

$$
\begin{aligned}
\frac{d}{d t}\|S\|^{2}+2 \int_{\Omega} S_{, i} S_{, i} d x & =-2 \int_{\Omega} S \omega_{i} C_{, i} d x+2 L \int_{\Omega} S\left(f(T)-f\left(T^{*}\right)\right) d x-2 k \int_{\Omega} S^{2} d x \\
& \leq 2 \int_{\Omega} S_{, i} \omega_{i} C d x+2 L k_{1} \int_{\Omega} S \theta d x \\
& \leq 2 \int_{\Omega} S_{, i} S_{, i} d x+\frac{\left(C^{M}\right)^{2}}{2}\|\omega\|^{2}+L^{2} k_{1}^{2}\|S\|^{2}+\|\theta\|^{2} .
\end{aligned}
$$

From (3.23), (3.24), and (3.26), we get

$$
\begin{aligned}
\frac{d}{d t}\left(\|\omega\|^{2}+\|\theta\|^{2}+\|S\|^{2}\right) \leq & \frac{2}{3} \lambda\left(\frac{3}{4} k_{2}^{2} k_{3}^{\frac{1}{2}} k_{4}^{\frac{3}{2}}+\frac{1}{4}|\Omega|\right) \\
& +\left(2+\frac{\left(T^{M}\right)^{2}}{2}+\frac{\left(C^{M}\right)^{2}}{2}\right)\|\omega\|^{2} \\
& +\left(L^{2} k_{1}^{2}+h^{2}\right)\|S\|^{2}+\left(1+g^{2}\right)\|\theta\|^{2} .
\end{aligned}
$$

We set

$$
F_{1}=\|\omega\|^{2}+\|\theta\|^{2}+\|S\|^{2} .
$$

Then we have

$$
\frac{d F_{1}}{d t} \leq \frac{2}{3} \lambda\left(\frac{3}{4} k_{2}^{2} k_{3}^{\frac{1}{2}} k_{4}^{\frac{3}{2}}+\frac{1}{4}|\Omega|\right)+M_{1} F_{1},
$$

where $M_{1}=\max \left\{2+\frac{\left(T^{M}\right)^{2}}{2}+\frac{\left(C^{M}\right)^{2}}{2}, L^{2} k_{1}^{2}+h^{2}, 1+g^{2}\right\}$.

We easily see that

$$
\|\omega\|^{2}+\|\theta\|^{2}+\|S\|^{2} \leq \frac{2 \lambda}{3 M_{1}}\left(\frac{3}{4} k_{2}^{2} k_{3}^{\frac{1}{2}} k_{4}^{\frac{3}{2}}+\frac{1}{4}|\Omega|\right) e^{M_{1} t} .
$$

Inequality (3.29) demonstrates the convergence of $u_{i}$ to $u_{i}^{*}, T$ to $T^{*}$, and $C$ to $C^{*}$ as $\lambda \rightarrow 0$ in the indicated measure.

Next, we will discuss the continuous dependence on the Forchheimer coefficient $\lambda$. Let $\left(u_{i}, p, T, C\right)$ be a solution of the boundary initial-value problem for the thermal convection 
model,

$$
\begin{aligned}
& \left\{\begin{array}{l}
\frac{\partial u_{i}}{\partial t}+\lambda_{1}|u| u_{i}=-p_{, i}+\Delta u_{i}+g_{i} T-h_{i} C, \quad(x, t) \in \Omega \times[0, \tau], \\
\frac{\partial u_{i}}{\partial x_{i}}=0, \quad(x, t) \in \Omega \times[0, \tau], \\
\frac{\partial T}{\partial t}+u_{i} \frac{\partial T}{\partial x_{i}}=\Delta T, \quad(x, t) \in \Omega \times[0, \tau], \\
\frac{\partial C}{\partial t}+u_{i} \frac{\partial C}{\partial x_{i}}=\Delta C+L f(T)-k C, \quad(x, t) \in \Omega \times[0, \tau],
\end{array}\right. \\
& u_{i}=0, \quad w \frac{\partial T}{\partial n}=0, \quad \frac{\partial C}{\partial n}=0, \quad(x, t) \in \partial \Omega \times[0, \tau], \\
& u_{i}(x, 0)=u_{i 0}(x), \\
& T(x, 0)=T_{0}(x), \quad C(x, 0)=C_{0}(x), \quad x \in \Omega .
\end{aligned}
$$

Furthermore, let $\left(u_{i}^{*}, p^{*}, T^{*}, C^{*}\right)$ be a solution to the following boundary initial-value problem:

$$
\begin{aligned}
& \left\{\begin{array}{l}
\frac{\partial u_{i}^{*}}{\partial t}+\lambda_{2}\left|u^{*}\right| u_{i}^{*}=-p_{, i}^{*}+\Delta u_{i}^{*}+g_{i} T^{*}-h_{i} C^{*}, \quad(x, t) \in \Omega \times[0, \tau], \\
\frac{\partial u_{i}^{*}}{\partial x_{i}}=0, \quad(x, t) \in \Omega \times[0, \tau], \\
\frac{\partial T^{*}}{\partial t}+u_{i}^{*} \frac{\partial T^{*}}{\partial x_{i}}=\Delta T^{*}, \quad(x, t) \in \Omega \times[0, \tau], \\
\frac{\partial C^{*}}{\partial t}+u_{i}^{*} \frac{\partial C^{*}}{\partial x_{i}}=\Delta C^{*}+L f\left(T^{*}\right)-k C^{*}, \quad(x, t) \in \Omega \times[0, \tau],
\end{array}\right. \\
& u_{i}^{*}=0, \quad \frac{\partial T^{*}}{\partial n}=0, \quad \frac{\partial C^{*}}{\partial n}=0, \quad(x, t) \in \partial \Omega \times[0, \tau], \\
& u_{i}^{*}(x, 0)=u_{i 0}(x), \\
& T^{*}(x, 0)=T_{0}(x), \quad C^{*}(x, 0)=C_{0}(x), \quad x \in \Omega .
\end{aligned}
$$

In this section, we establish the continuous dependence on the coefficient. To do this, let $\left(u_{i}, T, C, p\right)$ and $\left(u_{i}^{*}, T^{*}, C^{*}, p^{*}\right)$ be solutions of (3.30) and (3.34) with the same boundary and initial conditions. Now, we define

$$
\omega_{i}=u_{i}-u_{i}^{*}, \quad \pi=p-p^{*}, \quad \theta=T-T^{*}, \quad S=C-C^{*} .
$$

Then $\left(\omega_{i}, \theta, S, \pi\right)$ is a solution of the problem

$$
\left\{\begin{array}{l}
\frac{\partial \omega_{i}}{\partial t}+\left(\lambda_{1}|u| u_{i}-\lambda_{2}\left|u^{*}\right| u_{i}^{*}\right)=-\pi_{, i}+\Delta \omega_{i}+g_{i} \theta-h_{i} S, \quad(x, t) \in \Omega \times[0, \tau] \\
\frac{\partial \omega_{i}}{\partial x_{i}}=0, \quad(x, t) \in \Omega \times[0, \tau], \\
\frac{\partial \theta}{\partial t}+\omega_{i} \frac{\partial T}{\partial x_{i}}+u_{i}^{*} \frac{\partial \theta}{\partial x_{i}}=\Delta \theta, \quad(x, t) \in \Omega \times[0, \tau], \\
\frac{\partial S}{\partial t}+\omega_{i} \frac{\partial C}{\partial x_{i}}+u_{i}^{*} S_{, i}=\Delta S+L\left(f(T)-f\left(T^{*}\right)\right)-k S, \quad(x, t) \in \Omega \times[0, \tau],
\end{array}\right.
$$

in $\Omega \times t>0$, subject to the boundary and initial conditions

$$
\begin{aligned}
& \omega_{i}=0, \quad \frac{\partial \theta}{\partial n}=0, \quad \frac{\partial S}{\partial n}=0, \quad(x, t) \in \partial \Omega \times[0, \tau], \\
& \omega_{i}(x, 0)=0, \\
& \theta(x, 0)=0, \quad S(x, 0)=0, \quad x \in \Omega .
\end{aligned}
$$

We will obtain the following result. 
Theorem 2 Let $\left(u_{i}, T, C, p\right)$ be the classical solution to the initial-boundary problem (3.30)-(3.33), $\left(u_{i}^{*}, T^{*}, C^{*}, p^{*}\right)$ be the classical solution to the initial-boundary problem (3.34)-(3.37) in $\Omega \times(0, \tau)$, and $\left(w_{i}, \theta, S, \pi\right)$ be the difference of $\left(u_{i}, T, C, p\right)$ and $\left(u_{i}^{*}\right.$, $\left.T^{*}, C^{*}, p^{*}\right)$, then the solution $\left(u_{i}, T, C, p\right)$ converges to the solution $\left(u_{i}^{*}, T^{*}, C^{*}, p^{*}\right)$ as the Forchheimer coefficient $\lambda_{1}$ tends to $\lambda_{2}$. If we suppose that $\int_{\Omega} u_{i, t}(x, 0) u_{i, t}(x, 0) d x+$ $\int_{\Omega} T_{i, t}(x, 0) T_{i, t}(x, 0) d x+\int_{\Omega} C_{i, t}(x, 0) C_{i, t}(x, 0) d x \leq R$, the difference $\left(w_{i}, \theta, S, \pi\right)$ satisfies

$$
\|\omega\|^{2}+\|\theta\|^{2}+\|S\|^{2} \leq \lambda^{2} t e^{-M_{3} t} k_{2}^{2} k_{5}^{\frac{1}{2}} k_{6}^{\frac{3}{2}},
$$

where $\lambda=\lambda_{1}-\lambda_{2}$.

Proof We first observe that

$$
\frac{1}{2} \frac{d}{d t}\|\omega\|^{2}+\int_{\Omega}\left(\lambda_{1}|u| u_{i}-\lambda_{2}\left|u^{*}\right| u_{i}^{*}\right) \omega_{i} d x+\|\nabla \omega\|^{2}=\int_{\Omega} g_{i} \theta \omega_{i} d x-\int_{\Omega} h_{i} S \omega_{i} d x .
$$

Moreover, we can get

$$
\begin{aligned}
\int_{\Omega}\left(\lambda_{1}|u| u_{i}-\lambda_{2}\left|u^{*}\right| u_{i}^{*}\right) \omega_{i} d x & \geq\left(\lambda+\lambda_{2}\right) \int_{\Omega}|u| u_{i} \omega_{i} d x-\lambda_{2} \int_{\Omega}\left|u^{*}\right| u_{i}^{*} \omega_{i} d x \\
& \geq \lambda \int_{\Omega}|u| u_{i} \omega_{i} d x+\lambda_{2} \int_{\Omega}\left(|u| u_{i} \omega_{i}-\left|u^{*}\right| u_{i}^{*} \omega_{i}\right) d x .
\end{aligned}
$$

Since the operator $T(u)=|u| u$ is a monotonous operator, we get

$$
\int_{\Omega}\left(|u| u-\left|u^{*}\right| u^{*}\right) \omega d x \geq 0
$$

From the above discussion, we can get

$$
\int_{\Omega}\left(\lambda_{1}|u| u_{i}-\lambda_{2}\left|u^{*}\right| u_{i}^{*}\right) \omega_{i} d x \geq \lambda \int_{\Omega}|u| u_{i} \omega_{i} d x
$$

Hence we get a similar inequality,

$$
\frac{1}{2} \frac{d}{d t}\|\omega\|^{2}+\lambda \int_{\Omega}|u| u_{i} \omega_{i} d x+\|\nabla \omega\|^{2} \leq \int_{\Omega} g_{i} \theta \omega_{i} d x-\int_{\Omega} h_{i} S \omega_{i} d x
$$

Nevertheless, we use another method to get the bound for $\int_{\Omega}|u| u_{i} \omega_{i} d x$. We can use a similar method to give the bound for $\|u\|^{2}$,

$$
\|u\|^{2} \leq\left\|u_{0}\right\|^{2} e^{2 \tau}+\frac{1}{2}\left[g^{2}\left(T^{M}\right)^{2}+h^{2}\left(C^{M}\right)^{2}\right]|\Omega|\left(e^{2 \tau}-1\right)=k_{5} .
$$

The next step is to give a bound for $\|\nabla u\|^{2}$. In [27], Liu used the similar method. Multiplying $(1.1)_{1}$ by $u_{i}$ and integrating over $\Omega$, we have

$$
\begin{aligned}
\int_{\Omega} u_{i, j} u_{i, j} d x & \leq-\int_{\Omega} u_{i, t} u_{i} d x+\int_{\Omega} g_{i} u_{i} T d x-\int_{\Omega} h_{i} u_{i} C d x \\
& \leq\left(\int_{\Omega} u_{i, t} u_{i, t} d x\right)^{\frac{1}{2}}\left(\int_{\Omega} u_{i} u_{i} d x\right)^{\frac{1}{2}}+\left(\int_{\Omega} u_{i} u_{i} d x\right)^{\frac{1}{2}}\left(g^{2} \int_{\Omega} T^{2} d x\right)^{\frac{1}{2}}
\end{aligned}
$$




$$
\begin{aligned}
& +\left(\int_{\Omega} u_{i} u_{i} d x\right)^{\frac{1}{2}}\left(h^{2} \int_{\Omega} C^{2} d x\right)^{\frac{1}{2}} \\
\leq & k_{5}^{\frac{1}{2}}\left[\left(\int_{\Omega} u_{i, t} u_{i, t} d x\right)^{\frac{1}{2}}+|\Omega|^{\frac{1}{2}} g T^{M}+|\Omega|^{\frac{1}{2}} h C^{M}\right] .
\end{aligned}
$$

In order to have a bound for $\int_{\Omega} u_{i, j} u_{i, j} d x$, we need only give a bound for $\int_{\Omega} u_{i, t} u_{i, t} d x$. We can observe that

$$
\begin{aligned}
\frac{d}{d t} \int_{\Omega} u_{i, t} u_{i, t} d x= & 2 \int_{\Omega} u_{i, t}\left[-\lambda|u| u_{i}-p_{, i}+u_{i, j j}+g_{i} T-h_{i} C\right]_{, t} d x \\
\leq & -2 \lambda \int_{\Omega} u_{i, t}|u|, t u_{i} d x-2 \int_{\Omega} u_{i, j t} u_{i, j t} d x+2 \int_{\Omega} u_{i, t}\left(g_{i} T_{, t}-h_{i} C_{, t}\right) d x \\
\leq & -2 \lambda \int_{\Omega} u_{i, t} u_{i} \frac{u_{k} u_{k, t}}{|u|} d x+2 \int_{\Omega} u_{i, t} u_{i, t} d x+g^{2} \int_{\Omega} T_{, t} T_{, t} d x \\
& +h^{2} \int_{\Omega} C_{, t} C_{, t} d x \\
\leq & 2 \int_{\Omega} u_{i, t} u_{i, t} d x+g^{2} \int_{\Omega} T_{, t} T_{, t} d x+h^{2} \int_{\Omega} C_{, t} C_{, t} d x .
\end{aligned}
$$

Hence we should give the bound for $\int_{\Omega} T_{, t} T_{, t} d x$ and $\int_{\Omega} C_{, t} C_{, t} d x$. We find that

$$
\begin{aligned}
\frac{d}{d t} \int_{\Omega} T_{, t} T_{, t} d x & =2 \int_{\Omega} T_{, t}\left(T_{, i i t}-u_{i, t} T_{, i}-u_{i} T_{, i t}\right) d x=-2 \int_{\Omega} T_{, i t} T_{, i t} d x+2 \int_{\Omega} T_{, i t} u_{i, t} T d x \\
& \leq \frac{\left(T^{M}\right)^{2}}{2} \int_{\Omega} u_{i, t} u_{i, t} d x
\end{aligned}
$$

Similarly we can get

$$
\begin{aligned}
\frac{d}{d t} \int_{\Omega} C_{, t} C_{, t} d x= & 2 \int_{\Omega} C_{, t}\left(C_{, i i t}-u_{i, t} C_{, i}-u_{i} C_{, i t}+L[f(T)]_{, t}-k C_{, t}\right) d x \\
= & -2 \int_{\Omega} C_{, i t} C_{, i t} d x+2 \int_{\Omega} C_{, i t} u_{i, t} C d x \\
& +2 L \int_{\Omega} C_{, t}[f(T)]_{, t}-2 k \int_{\Omega} C_{, t} C_{, t} d x \\
\leq & \frac{\left(C^{M}\right)^{2}}{2} \int_{\Omega} u_{i, t} u_{i, t} d x+\frac{L^{2}}{2 k} \int_{\Omega}[f(T)]_{, t}[f(T)]_{, t} d x \\
\leq & \frac{\left(C^{M}\right)^{2}}{2} \int_{\Omega} u_{i, t} u_{i, t} d x+\frac{L^{2} k_{1}^{2}}{2 k} \int_{\Omega} T_{, t} T_{, t} d x
\end{aligned}
$$

We set

$$
F_{2}=\int_{\Omega} u_{i, t} u_{i, t} d x+\int_{\Omega} T_{, t} T_{, t} d x+\int_{\Omega} C_{, t} C_{, t} d x
$$

Combining (3.51)-(3.53), we can get

$$
\frac{d F_{2}}{d t} \leq M_{2} F_{2}
$$

where $M_{2}=\max \left\{2+\frac{\left(C^{M}\right)^{2}}{2}+\frac{\left(T^{M}\right)^{2}}{2}, \frac{L^{2} k_{1}^{2}}{2 k}+g^{2}, h^{2}\right\}$. 
Hence, from our assumption, we can get

$$
\begin{aligned}
F_{2} & \leq\left(\int_{\Omega} u_{i, t}(x, 0) u_{i, t}(x, 0) d x+\int_{\Omega} T_{i, t}(x, 0) T_{i, t}(x, 0) d x+\int_{\Omega} C_{i, t}(x, 0) C_{i, t}(x, 0) d x\right) e^{M_{2} t} \\
& \leq R e^{M_{2} \tau}
\end{aligned}
$$

So we can draw the conclusion that

$$
\int_{\Omega} u_{i, j} u_{i, j} d x \leq k_{5}^{\frac{1}{2}}\left(R e^{M_{2} \tau}+|\Omega|^{\frac{1}{2}} T^{M} g+|\Omega|^{\frac{1}{2}} C^{M} h\right)=k_{6} .
$$

Using the inequalities (3.16), we multiply $(3.39)_{1}$ by $\omega_{i}$ and integrate over $\Omega$ to find

$$
\begin{aligned}
\frac{d}{d t}\|\omega\|^{2}= & -2 \lambda \int_{\Omega}|u| u_{i} \omega_{i} d x-2\|\nabla \omega\|^{2} \\
& +2 \int_{\Omega} g_{i} \theta \omega_{i} d x-2 \int_{\Omega} h_{i} S \omega_{i} d x \\
\leq & \lambda^{2} \int_{\Omega}|u|^{4} d x+3\|\omega\|^{2}+g^{2}\|\theta\|^{2}+h^{2}\|S\|^{2} .
\end{aligned}
$$

From (3.24), (3.26), and (3.57), we get

$$
\begin{aligned}
\frac{d}{d t}\left(\|\omega\|^{2}+\|\theta\|^{2}+\|S\|^{2}\right) \leq & \lambda^{2} \int_{\Omega}|u|^{4} d x+\left(3+\frac{\left(T^{M}\right)^{2}}{2}+\frac{\left(C^{M}\right)^{2}}{2}\right)\|\omega\|^{2} \\
& +\left(L^{2} k_{1}^{2}+h^{2}\right)\|S\|^{2}+\left(1+g^{2}\right)\|\theta\|^{2} .
\end{aligned}
$$

We set

$$
F_{3}=\|\omega\|^{2}+\|\theta\|^{2}+\|S\|^{2}
$$

Then we have

$$
\frac{d F_{3}}{d t} \leq \lambda^{2} k_{2}^{2} k_{5}^{\frac{1}{2}} k_{6}^{\frac{3}{2}}+M_{3} F_{3}
$$

where $M_{3}=\max \left\{3+\frac{\left(T^{M}\right)^{2}}{2}+\frac{\left(C^{M}\right)^{2}}{2}, L^{2} k_{1}^{2}+h^{2}, 1+g^{2}\right\}$.

We easily see that

$$
\|\omega\|^{2}+\|\theta\|^{2}+\|S\|^{2} \leq \lambda^{2} t e^{-M_{3} t} k_{2}^{2} k_{5}^{\frac{1}{2}} k_{6}^{\frac{3}{2}} .
$$

Inequality (3.60) demonstrates the convergence of $u_{i}$ to $u_{i}^{*}, T$ to $T^{*}$, and $C$ to $C^{*}$ as $\lambda_{1} \rightarrow \lambda_{2}$ in the indicated measure.

\section{The case for the Forchheimer equations}

The above equations we discussed are of the Brinkman-Forchheimer equations type. If we consider the Forchheimer equations if $\Delta u$ is deleted, we will demonstrate another theorem. Now, let $\left(u_{i}, p, T, C\right)$ be a solution to the boundary initial-value problem for the 
Forchheimer model,

$$
\begin{aligned}
& \left\{\begin{array}{l}
\frac{\partial u_{i}}{\partial t}+\lambda|u| u_{i}=-p_{, i}+g_{i} T-h_{i} C, \quad(x, t) \in \Omega \times[0, \tau], \\
\frac{\partial u_{i}}{\partial x_{i}}=0, \quad(x, t) \in \Omega \times[0, \tau], \\
\frac{\partial T}{\partial t}+u_{i} \frac{\partial T}{\partial x_{i}}=\Delta T, \quad(x, t) \in \Omega \times[0, \tau], \\
\frac{\partial C}{\partial t}+u_{i} \frac{\partial C}{\partial x_{i}}=\Delta C+L f(T)-k C, \quad(x, t) \in \Omega \times[0, \tau],
\end{array}\right. \\
& u_{i}=0, \quad \frac{\partial T}{\partial n}=0, \quad \frac{\partial C}{\partial n}=0, \quad(x, t) \in \partial \Omega \times[0, \tau], \\
& u_{i}(x, 0)=u_{i 0}(x), \\
& T(x, 0)=T_{0}(x), \quad C(x, 0)=C_{0}(x), \quad x \in \Omega .
\end{aligned}
$$

Furthermore, let $\left(u_{i}^{*}, p^{*}, T^{*}, C^{*}\right)$ be a solution to the following boundary initial-value problem:

$$
\begin{aligned}
& \left\{\begin{array}{l}
\frac{\partial u_{i}^{*}}{\partial t}=-p_{, i}^{*}+g_{i} T^{*}-h_{i} C^{*}, \quad(x, t) \in \Omega \times[0, \tau], \\
\frac{\partial u_{i}^{*}}{\partial x_{i}}=0, \quad(x, t) \in \Omega \times[0, \tau], \\
\frac{\partial T^{*}}{\partial t}+u_{i}^{*} \frac{\partial T^{*}}{\partial x_{i}}=\Delta T^{*}, \quad(x, t) \in \Omega \times[0, \tau], \\
\frac{\partial C^{*}}{\partial t}+u_{i}^{*} \frac{\partial C^{*}}{\partial x_{i}}=\Delta C^{*}+L f\left(T^{*}\right)-k C^{*}, \quad(x, t) \in \Omega \times[0, \tau],
\end{array}\right. \\
& u_{i}^{*}=0, \quad \frac{\partial T^{*}}{\partial n}=0, \quad \frac{\partial C^{*}}{\partial n}=0, \quad(x, t) \in \partial \Omega \times[0, \tau], \\
& u_{i}^{*}(x, 0)=u_{i 0}(x), \\
& T^{*}(x, 0)=T_{0}(x), \quad C^{*}(x, 0)=C_{0}(x), \quad x \in \Omega .
\end{aligned}
$$

In this section, we establish convergence on the coefficient $\lambda$. To do this, let $\left(u_{i}, T, C, P\right)$ and $\left(u_{i}^{*}, T^{*}, C^{*}, P^{*}\right)$ be solutions of (4.1) and (4.5) with the same boundary and initial conditions. Now we define

$$
\omega_{i}=u_{i}-u_{i}^{*}, \quad \pi=p-p^{*}, \quad \theta=T-T^{*}, \quad S=C-C^{*} .
$$

Then $\left(\omega_{i}, \theta, S, \pi\right)$ is a solution of the problem

$$
\left\{\begin{array}{l}
\frac{\partial \omega_{i}}{\partial t}+\lambda|u| u_{i}=-\pi_{, i}+g_{i} \theta-h_{i} S, \quad(x, t) \in \Omega \times[0, \tau] \\
\frac{\partial \omega_{i}}{\partial x_{i}}=0, \quad(x, t) \in \Omega \times[0, \tau], \\
\frac{\partial \theta}{\partial t}+\omega_{i} \frac{\partial T}{\partial x_{i}}+u_{i}^{*} \frac{\partial \theta}{\partial x_{i}}=\Delta \theta, \quad(x, t) \in \Omega \times[0, \tau], \\
\frac{\partial S}{\partial t}+\omega_{i} \frac{\partial C}{\partial x_{i}}+u_{i}^{*} S_{, i}=\Delta S+L\left(f(T)-f\left(T^{*}\right)\right)-k S, \quad(x, t) \in \Omega \times[0, \tau]
\end{array}\right.
$$

in $\Omega \times[0, \tau]$, subject to the boundary and initial conditions

$$
\begin{aligned}
& \omega_{i}=0, \quad \frac{\partial \theta}{\partial n}=0, \quad \frac{\partial S}{\partial n}=0, \quad(x, t) \in \partial \Omega \times[0, \tau], \\
& \omega_{i}(x, 0)=0, \\
& \theta(x, 0)=0, \quad S(x, 0)=0, \quad x \in \Omega .
\end{aligned}
$$

We will obtain the following result. 
Theorem 3 Let $\left(u_{i}, T, C, p\right)$ be the classical solution to the initial-boundary problem (4.1)(4.4), $\left(u_{i}^{*}, T^{*}, C^{*}, p^{*}\right)$ be the classical solution to the initial-boundary problem (4.5)-(4.8) in $\Omega \times(0, \tau)$, and $\left(w_{i}, \theta, S, \pi\right)$ be the difference of $\left(u_{i}, T, C, p\right)$ and $\left(u_{i}^{*}, T^{*}, C^{*}, p^{*}\right)$, then the solution $\left(u_{i}, T, C, p\right)$ converges to the solution $\left(u_{i}^{*}, T^{*}, C^{*}, p^{*}\right)$ as the Forchheimer coefficient $\lambda$ tends to 0 . The difference $\left(w_{i}, \theta, S, \pi\right)$ satisfies

$$
\|\omega\|^{2}+\|\theta\|^{2}+\|S\|^{2} \leq \lambda^{2} t e^{-M_{3} t} k_{2}^{2} k_{5}^{\frac{1}{2}} k_{7}^{\frac{3}{2}} .
$$

We can also get the continuous dependence result for different Forchheimer coefficients $\lambda \rightarrow 0$.

Proof First of all, we may calculate $\int_{0}^{t} \int_{\Omega} C_{, i} C_{, i} d x d \eta$ and $\int_{0}^{t} \int_{\Omega} T_{, i} T_{, i} d x d \eta$,

$$
\begin{aligned}
\frac{\partial}{\partial t} \int_{\Omega} C^{2} d x & =2 \int_{\Omega} C\left(\Delta C+L f(T)-k C-u_{i} C_{, i}\right) d x \\
& =-2 \int_{\Omega} C_{, i} C_{, i} d x+2 L \int_{\Omega} C f(T) d x-2 k \int_{\Omega} C^{2} d x \\
& \leq-2 \int_{\Omega} C_{, i} C_{, i} d x+\frac{L^{2} d^{2}}{2 k}|\Omega| .
\end{aligned}
$$

Integrating (4.15) over $\Omega$, we find

$$
2 \int_{0}^{t} \int_{\Omega} C_{, i} C_{, i} d x d \eta \leq \int_{\Omega} C_{0}^{2} d x+\frac{1}{2 k} L^{2} d^{2} \tau|\Omega| .
$$

Similarly, we can obtain

$$
2 \int_{0}^{t} \int_{\Omega} T_{, i} T_{, i} d x d \eta \leq \int_{\Omega} T_{0}^{2} d x
$$

Then we want to give a bound for $\|\nabla u\|^{2}$. We know that

$$
\int_{\Omega} u_{i, j} u_{i, j} d x=\int_{\Omega} u_{i, j}\left(u_{i, j}-u_{j, i}\right) d x+\int_{\Omega} u_{i, j} u_{j, i} d x
$$

After some integration by parts, it implies

$$
\begin{aligned}
\int_{\Omega} u_{i, j} u_{j, i} d x & =\oint_{\partial \Omega} u_{i, j} u_{j} n_{i} d s-\int_{\Omega} u_{i, i j} u_{j} d x \\
& =-\oint_{\partial \Omega} u_{i, i} u_{j} n_{j} d s+\int_{\Omega} u_{i, i} u_{j, j} d x=0 .
\end{aligned}
$$

We set

$$
J(t)=\int_{\Omega} u_{i, j}\left(u_{i, j}-u_{j, i}\right) d x .
$$

We note that

$$
k_{g}^{2}=\max _{\Omega} g_{i, j} g_{i, j}, \quad k_{h}^{2}=\max _{\Omega} h_{i, j} h_{i, j}
$$


We can obtain

$$
\begin{aligned}
\frac{d J}{d t}= & 2 \int_{\Omega} u_{i, j t} u_{i, j} d x-\int_{\Omega} u_{i, j t} u_{j, i} d x-\int_{\Omega} u_{j, i} u_{i, j} d x \\
= & 2 \int_{\Omega} u_{i, j t}\left(u_{i, j}-u_{j, i}\right) d x \\
= & 2 \int_{\Omega}\left(u_{i, j}-u_{j, i}\right)\left[-\lambda\left(|u| u_{i}\right)_{, j}-p_{i, j}+\left(g_{i} T\right)_{, j}-\left(h_{i} C\right)_{, j}\right] d x \\
= & -2 \lambda \int_{\Omega}|u| u_{i, j} u_{i, j} d x-2 \lambda \int_{\Omega} u_{i, j} u_{i} \frac{u_{k} u_{k, j}}{|u|} d x+2 \lambda \oint_{\partial \Omega} u_{j, i}|u| u_{i} n_{j} d s \\
& +2 \int_{\Omega}\left(u_{i, j}-u_{j, i}\right)\left(g_{i, j} T-h_{i, j} C\right) d x+2 \int_{\Omega}\left(u_{i, j}-u_{j, i}\right)\left(g_{i} T_{, j}-h_{i} C_{, j}\right) d x \\
\leq & 2 \int_{\Omega}\left(u_{i, j}-u_{j, i}\right)\left(u_{i, j}-u_{j, i}\right) d x+2 k_{m}^{2} \int_{\Omega}\left(T^{2}+C^{2}\right) d x \\
& +2 k_{n}^{2} \int_{\Omega}\left(T_{, j} T_{, j}+C_{, j} C_{, j}\right) d x,
\end{aligned}
$$

where $k_{m}^{2}=\max \left\{k_{g}^{2}, k_{h}^{2}\right\}, k_{n}^{2}=\max \left\{g^{2}, h^{2}\right\}$.

We know

$$
\int_{\Omega}\left(u_{i, j}-u_{j, i}\right)\left(u_{i, j}-u_{j, i}\right) d x=2 \int_{\Omega}\left(u_{i, j}-u_{j, i}\right) u_{i, j} d x=2 J(t) .
$$

From (4.22) and (4.23), we can get

$$
\frac{d J}{d t} \leq 4 J+2 k_{m}^{2}|\Omega|\left[\left(T^{M}\right)^{2}+\left(C^{M}\right)^{2}\right]+2 k_{n}^{2} \int_{\Omega}\left(T_{, j} T_{, j}+C_{, j} C_{, j}\right) d x .
$$

Combining (4.16), (4.17), and (4.24), we have

$$
\begin{aligned}
\|\nabla u\|^{2} \leq & 2 k_{m}^{2} e^{4 \tau}|\Omega| \tau\left[\left(T^{M}\right)^{2}+\left(C^{M}\right)^{2}\right] \\
& +e^{4 \tau} k_{n}^{2}\left(\int_{\Omega} T_{0}^{2}+C_{0}^{2} d x+\frac{1}{2 k} L^{2} d^{2} \tau|\Omega|\right) \\
= & k_{7} .
\end{aligned}
$$

Similarly we can obtain

$$
\|u\|_{4}^{4} \leq k_{2}^{2} k_{5}^{\frac{1}{2}} k_{7}^{\frac{3}{2}}
$$

We can use a similar method to get the result that

$$
\|\omega\|^{2}+\|\theta\|^{2}+\|S\|^{2} \leq \lambda^{2} t e^{-M_{3} t} k_{2}^{2} k_{5}^{\frac{1}{2}} k_{7}^{\frac{3}{2}} .
$$

In inequalities (4.27) we demonstrate the convergence of $u_{i}$ to $u_{i}^{*}, T$ to $T^{*}$, and $C$ to $C^{*}$ as $\lambda \rightarrow 0$ in the indicated measure. We can also get the continuous dependence result. 
Authors' contributions

The authors declare that the study was realized in collaboration with the same responsibility. All authors read and approved the final manuscript.

\section{Acknowledgements}

The authors would like to express their gratitude to the anonymous referees for helpful and very careful reading on this paper. The work was supported by the national natural Science Foundation of China (Grant No. 11201087), Foundation for Technology Innovation in Higher Education of Guangdong, China (Grant No. 2013KJCX0136), the Excellent Young Teachers Training Program for colleges and universities of Guangdong Province, China (Grant No. Yq2013121), the Guangdong college students' innovation of science and technology cultivate the special funds, and the innovation and strength project for university in Guangdong Province.

\section{Received: 31 August 2015 Accepted: 7 February 2016 Published online: 26 February 2016}

\section{References}

1. Ames, KA, Straughan, B: Non-standard and Improperly Posed Problems. Mathematics in Science and Engineering, vol. 194. Academic press, San Diego (1997)

2. Nield, DA, Bejan, A: Convection in Porous Media. Springer, New York (1992)

3. Straughan, B: The Energy Method, Stability and Nonlinear Convection, 2nd edn. Applied Mathematical Sciences, vol. 91. Springer, Berlin (2004)

4. Straughan, B: Stability and Wave Motion in Porous Media. Applied Mathematical Sciences, vol. 165. Springer, Berlin (2008)

5. Ames, KA, Payne, LE: On stabilizing against modelling errors in a penetrative convection problem for a porous medium. Math. Models Methods Appl. Sci. 4, 733-740 (1994)

6. Franchi, F, Straughan, B: Continuous dependence and decay for the Forchheimer equations. Proc. R. Soc. Lond. A 459, 3195-3202 (2003)

7. Kaloni, PN, Qin, Y: Steady nonlinear double-diffusive convection in a porous medium base upon the Brinkman-Forchheimer model. J. Math. Anal. Appl. 204, 138-155 (1996)

8. Kaloni, PN, Guo, J: Steady nonlinear double-diffusive convection in a porous medium based upon the Brinkman-Forchheimer model. J. Math. Anal. Appl. 204, 138-155 (1996)

9. Payne, LE, Straughan, B: Stability in the initial-time geometry problem for the Brinkman and Darcy equations of flow in porous media. J. Math. Pures Appl. 75, 255-271 (1996)

10. Payne, LE, Straughan, B: Convergence and continuous dependence for the Brinkman-Forchheimer equations. Stud. Appl. Math. 102, 419-439 (1999)

11. Payne, LE, Straughan, B: Structural stability for the Darcy equations of flow in porous media. Proc. R. Soc. Lond. A 454, 1691-1698 (1998)

12. Payne, LE, Song, JC, Straughan, B: Continuous dependence and convergence results for Brinkman and Forchheimer models with variable viscosity. Proc. R. Soc. Lond. A 455, 2173-2190 (1999)

13. Lin, C, Payne, LE: Structural stability for a Brinkman fluid. Math. Methods Appl. Sci. 30, 567-578 (2007)

14. Lin, C, Payne, LE: Structural stability for the Brinkman equations of flow in double diffusive convection. J. Math. Anal. Appl. 325, 1479-1490 (2007)

15. Lin, C, Payne, LE: Continuous dependence on the Soret coefficient for double diffusive convection in Darcy flow. J. Math. Anal. Appl. 342, 311-325 (2008)

16. Li, Y, Lin, C: Continuous dependence for the nonhomogeneous Brinkman-Forchheimer equations in a semi-infinite pipe. Appl. Math. Comput. 244, 201-208 (2014)

17. Celebi, AO, Kalantarov, VK, Ugurlu, D: On continuous dependence on coefficients of the Brinkman-Forchheimer equations. Appl. Math. Lett. 19, 801-807 (2006)

18. Celebi, AO, Kalantarov, VK, Ugurlu, D: Continuous dependence for the convective Brinkman-Forchheimer equations Appl. Anal. 84, 877-888 (2005)

19. Straughan, B: Continuous dependence on the heat source in resonant porous penetrative convection. Stud. Appl. Math. 127, 302-314 (2011)

20. Scott, NL: Continuous dependence on boundary reaction terms in a porous medium of Darcy type. J. Math. Anal. Appl. 399, 667-675 (2013)

21. Scott, NL, Straughan, B: Continuous dependence on the reaction terms in porous convection with surface reactions. Q. Appl. Math. 71, 501-508 (2013)

22. Harfash, AJ: Continuous dependence on the coefficients for double diffusive convection in Darcy flow with magnetic field effect. Anal. Math. Phys. 3, 163-181 (2013)

23. Harfash, AJ: Structural stability for convection models in a reacting porous medium with magnetic field effect. Ric. Mat. 63, 1-13 (2014)

24. Harfash, AJ: Structural stability for two convection models in a reacting fluid with magnetic field effect. Ann. Henri Poincaré 15, 2441-2465 (2014)

25. Ciarletta, M, Straughan, B, Tibullo, V: Structural stability for a thermal convection model with temperature-dependent solubility. Nonlinear Anal., Real World Appl. 22, 34-43 (2015)

26. Fife, PC: A gentle introduction to the physics and mathematics of incompressible flow. www.math.utah.edu/ fife/gentleb.pdf (2000)

27. Liu, Y: Convergence results for Forchheimer's equations. Eur. J. Appl. Math. 23, 761-775 (2012) 